\title{
Sensor Technologies for Particulate Detection and Characterization
}

\author{
Paul S. Greenberg \\ NASA-Glenn Research Center \\ Paul.S.Greenberg@nasa.gov
}

\begin{abstract}
Planned Lunar missions have resulted in renewed attention to problems attributable to fine particulates. While the difficulties experienced during the sequence of Apollo missions did not prove critical in all cases, the comparatively long duration of impending missions may present a different situation.

This situation creates the need for a spectrum of particulate sensing technologies. From a fundamental perspective, an improved understanding of the properties of the dust fraction is required. Described here is laboratory-based reference instrumentation for the measurement of fundamental particle size distribution (PSD) functions from 2.5 nanometers to 20 micrometers. Concomitant efforts for separating samples into fractional size bins are also presented.

A requirement also exists for developing mission compatible sensors. Examples include provisions for air quality monitoring in spacecraft and remote habitation modules. Required sensor attributes such as low mass, volume, and power consumption, autonomy of operation, and extended reliability cannot be accommodated by existing technologies.
\end{abstract}

\section{Introduction}

The NASA exploration program defines planetary missions of unprecedented durations. In response, a renewed attention to problems attributable to fine particulates has been initiated. Tours of duty for individual crew members, for example, are anticipated to span periods on the order of six months. In addition, many hardware elements will be required to operate continuously in the Lunar and Martian surface environments for time frames of years. As such, a combination of suitably tolerant system elements in combination with effective mitigation strategies are now required.

Addressing these issues requires a variety of technologies for the detection and characterization of fine (100 nanometers - 10 micrometers) and ultrafine $(<100$ nanometers) particulates. From a fundamental perspective, an improved understanding of 
the properties of the dust fraction of surface regolith is required. Prior studies of the micron to millimeter fraction demonstrate correlations between the particles various structural and compositional properties and their size. This relationship may persist into the submicron regime, affecting critical properties such as hardness, abrasivity, surface reactivity, and magnetic susceptibility. Therefore, characterizing samples at the submicron level on the basis of particle size is essential. The effort described here includes the specification, calibration, and utilization of laboratory-based reference instrumentation for the measurement of fundamental particle size distribution (PSD) functions continuously spanning the range from 2.5 nanometers to 10 micrometers. This size specification addresses what is effectively Lunar "dust," i.e. the characteristic range where electrostatic and Van der Waals dominate inertial effects. The same values are specified for the nominal respirable range, corresponding to particulate sizes attributable to observed human respiratory health effects. Concomitant efforts for separating samples into fractional size bins are also described. Present activities include high-resolution differentiation and substrate deposition, as well as provisions for the coarser differentiation of materials in bulk quantity. These same capabilities are required to support the iterative development and refinement of suitable simulant materials.

A requirement also exists for end-use, mission compatible sensor technologies. In the context of Lunar Exploration, examples include provisions for air quality monitoring in spacecraft and remote habitation modules, as well as process control monitors for associated filtration systems. Required sensor attributes such as low mass, volume, and power consumption, autonomy of operation, and extended reliability cannot be accommodated by existing technologies. For these reasons, an effort exists within NASA to develop advanced sensor technologies to address these requirements. This development leverages from synergistic activities involving NASA sensor development for advanced spacecraft fire detection, and terrestrial field applications sponsored by other Federal Agencies involving human respiratory exposure to particulates. A variety of approaches are being pursued to address the required range of anticipated particle sizes and concentrations. The progress of these efforts must also reflect ongoing refinements with NASA and the external community insofar as size-dependent performance metrics and dosimetry standards are concerned.

\section{Reference Measurements of Particle Size Distributions}

Advancing our fundamental understanding of Lunar regolith involves the basic measurement of particle size distributions (PSDs). This is particularly true for the nominal dust portion; i.e. sizes ranging from 10 micrometers and below. The same requirement arises for the production, characterization, and refinement of regolith simulants. In the latter case, it is desirable from a variety of perspectives to replicate the associated PSD of actual Lunar material. Further, the approach of constructing simulants from a collection of sub-components requires characterizing the PSDs of the building blocks as well as that of the composite mixture. The unique nature of regolith formation has been observed to produce structural and compositional features within larger fractions that correlate with particle size, ${ }^{1}$ and it is possible that this situation will persist into the dust regime. One such example is the $\mathrm{Is} / \mathrm{FeO}$ ratio, sometimes referred to as the 
soil maturity index. ${ }^{2}$ As such, it is also desirable to sort and isolate material by size in the fine and ultrafine ranges for latter analysis by other methods. The class of techniques utilized here are based on aerodynamic diameter: the diameter of a sphere of unit density that has the same gravitational settling velocity as the particle in question. While a number of definitions exist for the specification of particle size, the aerodynamic diameter is useful in this context since it is most closely related to the associated transport properties.

A variety of experimental methods have been developed by the aerosol science community for this measurement, many of which are mature and utilized both widely and routinely. In this case, submicron particles are measured using a Differential Mobility Analyzer (DMA). ${ }^{3}$ The DMA directly measures a quantity related to the aerodynamic diameter, the particle electrical mobility, $\mathrm{Zp}$ :

$$
\mathrm{Z}_{\mathrm{p}}=\frac{\mathrm{n}_{\mathrm{e}} \mathrm{C}_{\mathrm{c}}(\mathrm{Kn})}{3 \pi \eta \mathrm{D}_{\mathrm{p}}}
$$

where $n_{e}$ is the number of electrical charges carried by a particle of diameter $D_{p}, C_{c}(K n)$ is the Cunningham slip factor that accounts for noncontinuum effects when the particle size becomes comparable with or smaller than the mean free path of the surrounding gas, and $\eta$ is the dynamic viscosity of the surrounding gas. The slip factor is a function of the Knudsen number , $K n=2 \lambda / D_{p}$, where $\lambda$ is the mean free path of the gas molecules. If a known charge, $\mathrm{n}_{\mathrm{e}}$, is applied, the particle diameter is determined by measuring the ratio of viscous to electrostatic forces. The most common configuration classifies $Z_{p}$ by differences in the resulting spatial trajectories ${ }^{4}$, and the requisite charge states are achieved by either isotope diffusion or unipolar corona charging. ${ }^{5}$ Having been sorted on the basis of electrical mobility, the number of particles occurring in a given size bin are individually counted using a Condensate Particle Counter (CPC). ${ }^{3}$

Supermicron particles are measured using an Aerodynamic Particle Sizer (APS). The APS determines the aerodynamic transport diameter by measuring the time-of-flight (TOF) of particles entrained in an accelerating gas flow. ${ }^{6}$ The resulting lag between the gas and particle velocities, respectively, is a function of the particle diameter. TOF of individual particles is measured by a pair of laser beams axially focused on the exit region of the acceleration nozzle. Light scattered by the particles is collected by an avalanche photodiode, and the observed spacing between the two optical pulses is used to accurately measure the transit time through the optical sample volume. The observed velocity lag is independent of particle density within the Stokes regime.

Particle samples can also be acquired for subsequent analysis or SEM interrogation using an Electrically-assisted Aerosol Sampler (EAS). ${ }^{7}$ The EAS deposits the aerosolized sample on various substrate materials. It is especially useful in tandem with a DMA. In this case, the DMA can be parked or "tuned" to a specific size bin, with the collected sample material representing only that narrow slice of the total population. 
A schematic of the overall measurement configuration is shown in figure 1 . The apparatus also includes a Small Sample Particle Disperser ${ }^{8}$ for aerosolizing the dry material. A personal computer is used to scan the DMA through its size range, and record the resulting data stream from the CPC and APS.

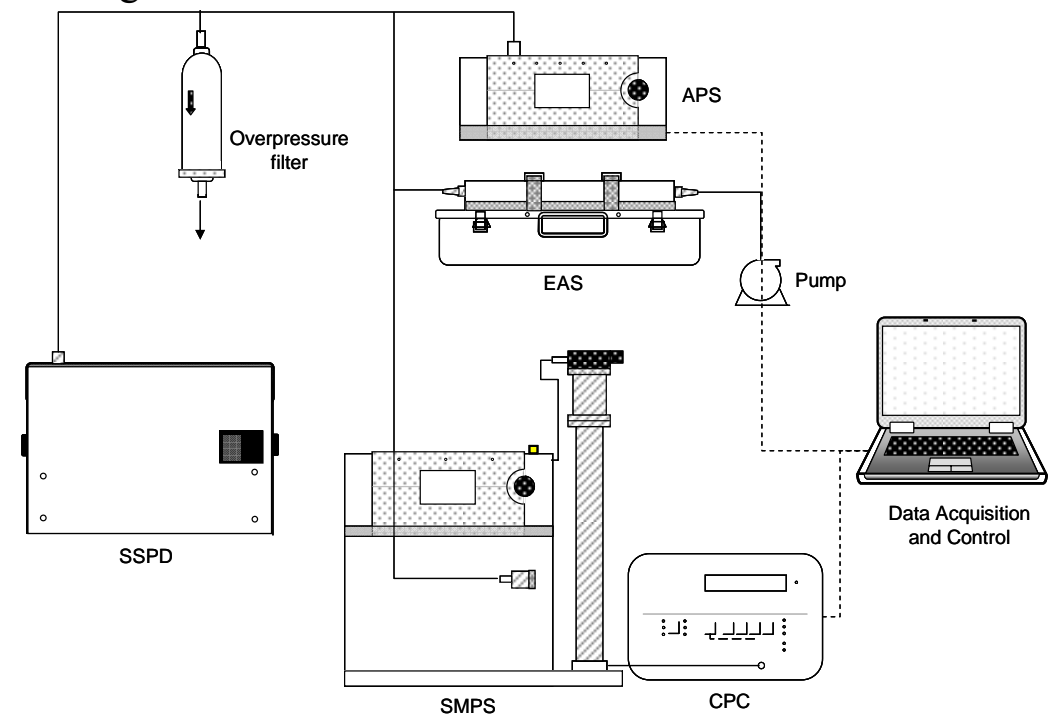

Figure 1: Experimental configuration for PSD measurements

Shown in figure 2 is composite PSD data obtained with this combination of instruments. The measurements correspond to two regolith simulants; JSC1Af and a new candidate material presently under evaluation. The counts per bin were normalized to the peak value for comparison. Both samples were mechanically sieved at the 20 micrometer level prior to analysis.

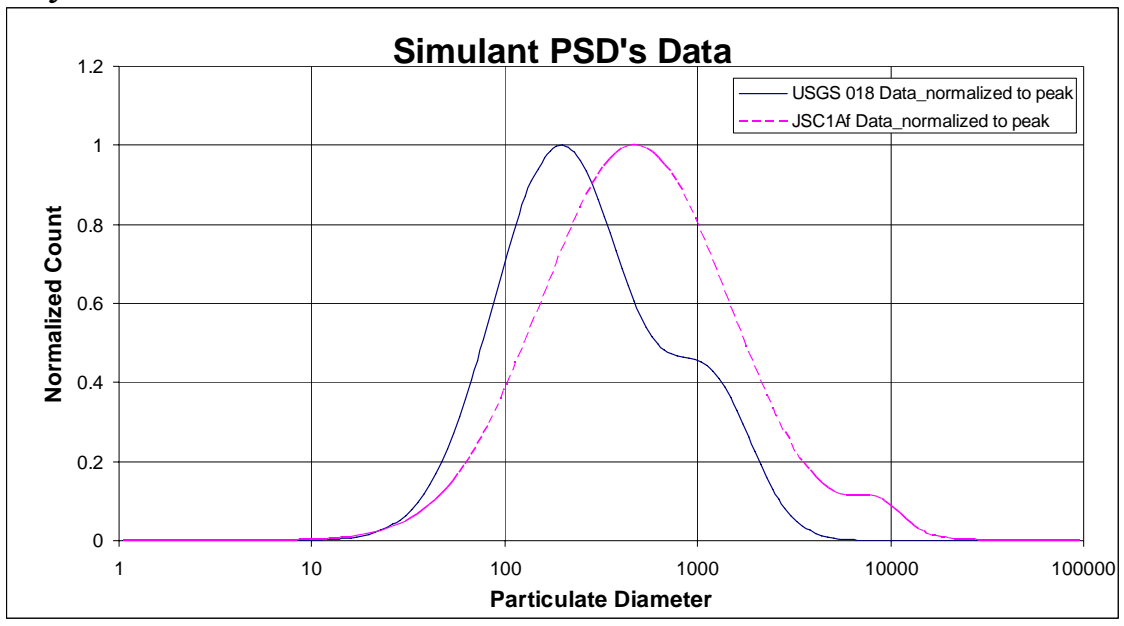

Figure 2: Normalized PSDs for JSC1AF and USGS NU-LHT-1M 018

Figure 3 illustrates two size fractions of the second simulant that were separated with a tuned DMA as previously described. 

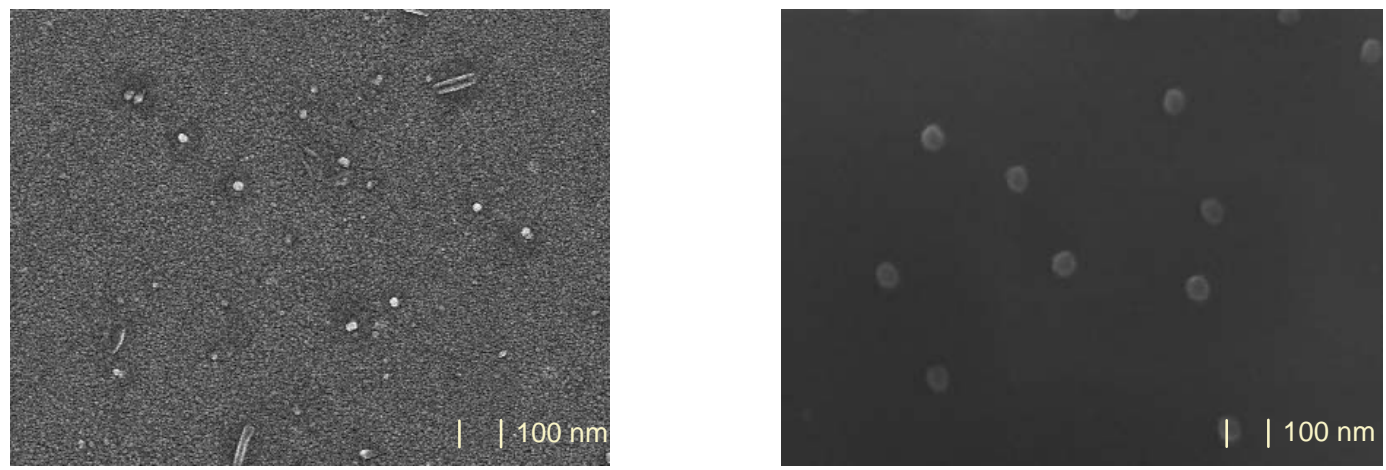

Figure 3: Size fractioned samples using a DMA. The left and right panels show SEM images of $20 \mathrm{~nm}$ and $50 \mathrm{~nm}$ fractions, respectively.

\section{Cyclonic Separation of Bulk Material}

In some cases, it is desirable to separate comparatively large quantities of material in narrow size fractions. The resulting material can then be used to conduct subsequent analysis of size-dependent properties or for post-engineering (e.g. remixing) of simulant mixtures to obtain desired geotechnical properties.

To this end, a cyclone separator was designed, built, and tested. The complete unit provides six size bins as shown in figure 4. As illustrated, the two smallest bins (100 and $20 \mathrm{~nm}$ ) must be operated at reduced pressure. Physically, this is required to reduce the ratio of viscous to inertial forces necessary in maximizing the steepness of the size cutoff

characteristics.

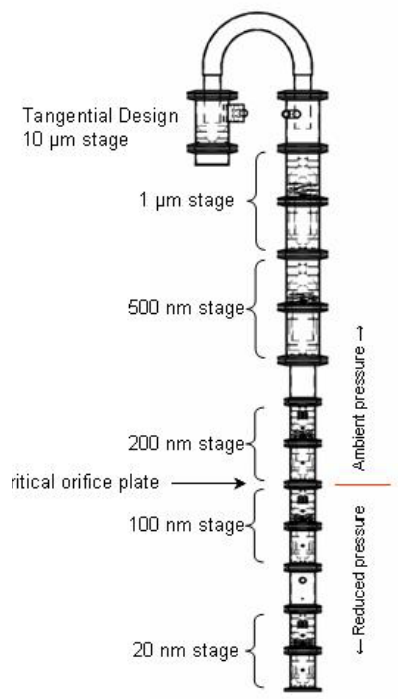

Figure 4: Six stage cyclone separator
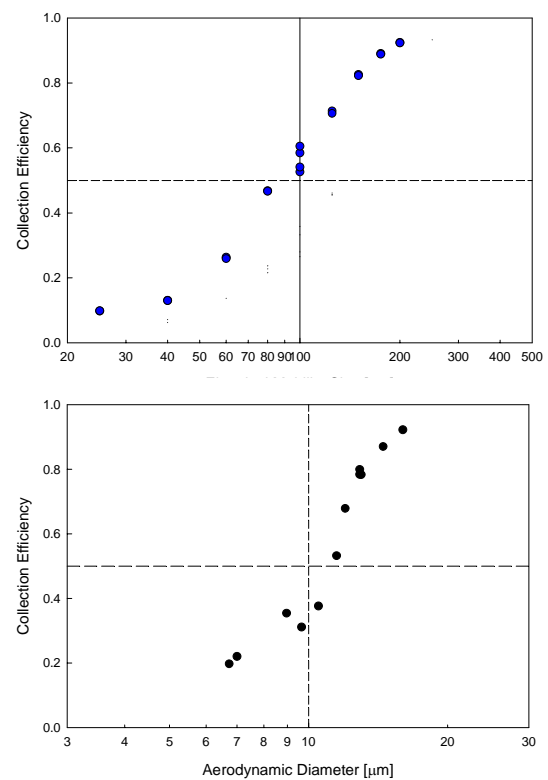

Figure 5: Cutoff curves showing $d_{50}$ for stages one $(10 \mu \mathrm{m})$ and five $(100 \mathrm{~nm})$.

Figure 5 illustrates $\mathrm{d}_{50}$, the cutoff diameter at $50 \%$ collection efficiency for stages one and five. The present effort concerns the construction of a high output aerosolizer to supply material to the cyclone input. The performance goal is on the order of $\mathrm{kg} / \mathrm{day}$ of bulk material.

\section{Advanced Sensor Technologies for Environmental Monitoring}


The eventual establishment of human habitats on the Lunar surface will pose the need for particulate environmental monitors. This situation has lead to the establishment of draft dosimetry values, ${ }^{9}$ and the recognition that suitable sensor technologies will be required. Because the associated features of mission deployment, i.e. size, mass, power consumption, reliability and autonomy of operation, cannot be met by existing technologies, a nominal project has been proposed to address this gap. A low level effort is provided at present due to the comparatively long timetable for delivery, so the results described here are attributable to other synergistic activities. These efforts relate to terrestrial applications for environmental monitoring and human health effects.

Resolving submicron PSDs follows a similar approach to that described in Section II. Specifically, a miniature DMA has been developed and tested. ${ }^{10}$ A photograph of the prototype Disc Classifier is shown in figure 6. The total volume of the device is

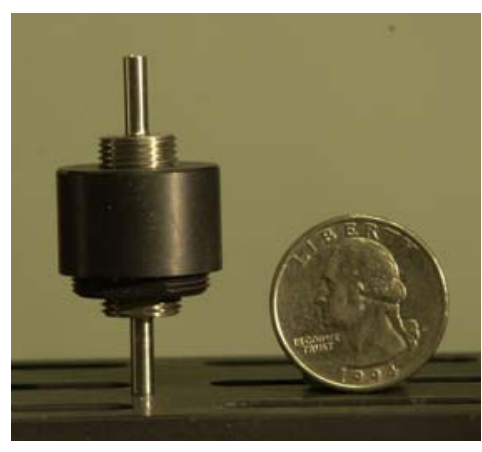

Figure 6: Prototype minintura diso nak

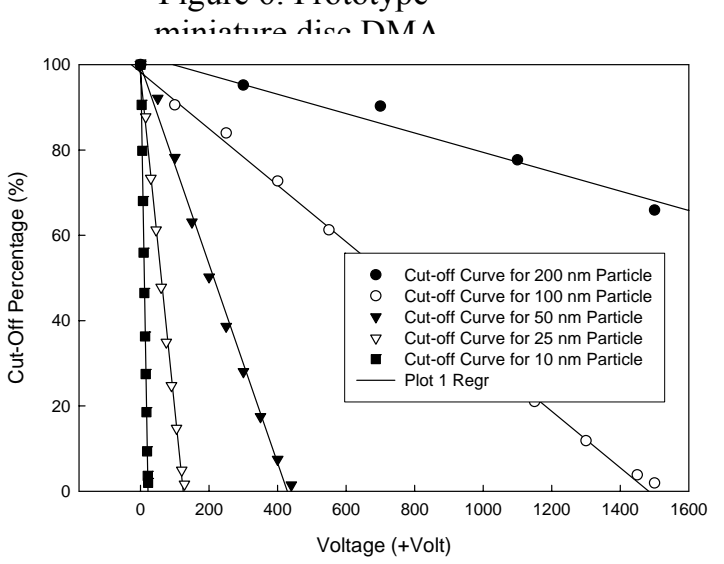

Figure 7: DMA cutoff curves for various particle sizes approximately $3 \mathrm{~cm}^{3}$, compared with the benchtop size of the commercial Scanning Mobility Particle Sizer (SMPS) shown in figure 1 . The characteristic cutoff curves displaying the percent penetration vs. applied voltage for various particle sizes is shown in figure 7 . This behavior is shown in reduced coordinates of $\mathrm{Zp}$, the particle electrical mobility, in figure 8 . Also seen is the excellent agreement between modeled and observed performance. While the shorter internal trajectories provide somewhat decreased resolution in mobility space relative to the commercial SMPS, internal losses are also decreased.

As discussed, mobility classification requires the establishment of known electrical charge states on the incoming particle sample. Although diffusion charging using an isotope source is commonly used for reasons of simplicity, it is extremely inefficient. This is particularly the case in the nanometer regime. Residual neutrals are not classified, as seen in eqn. (1) for the case of $n_{e}=0$. Detection efficiency becomes increasingly important as the sensor dimensions decrease, since the particle flux is essentially proportional to the square of the characteristic dimensions of the device. 
For this reason, a miniature unipolar field charger was developed for use in tandem with the small disc DMA. ${ }^{11}$ A photograph of this device is shown in figure 9. The resulting charging efficiency is provided in figure 10. The figure of merit is the extrinsic efficiency, which accounts for internal losses within the device. As seen, a more than ten-fold increase in efficiency is achieved relative to isotope diffusion charging [BipolarFuchs]. Advances in the materials and geometry of the discharge electrodes allow the device to be operated at modest voltages ( $\sim 40 \mathrm{~V} \max )$, in comparison with the $\mathrm{kV}$ requirements of previously demonstrated unipolar configurations.

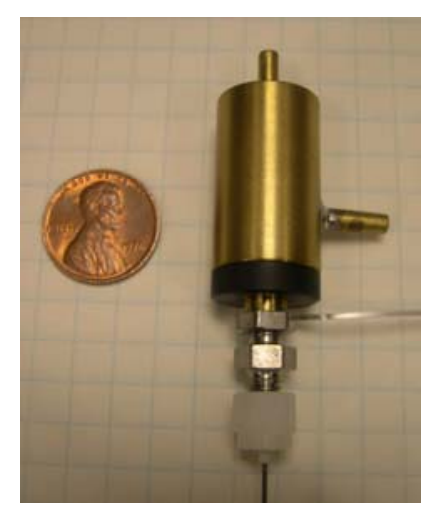

Figure 9: Miniature unipolar charger

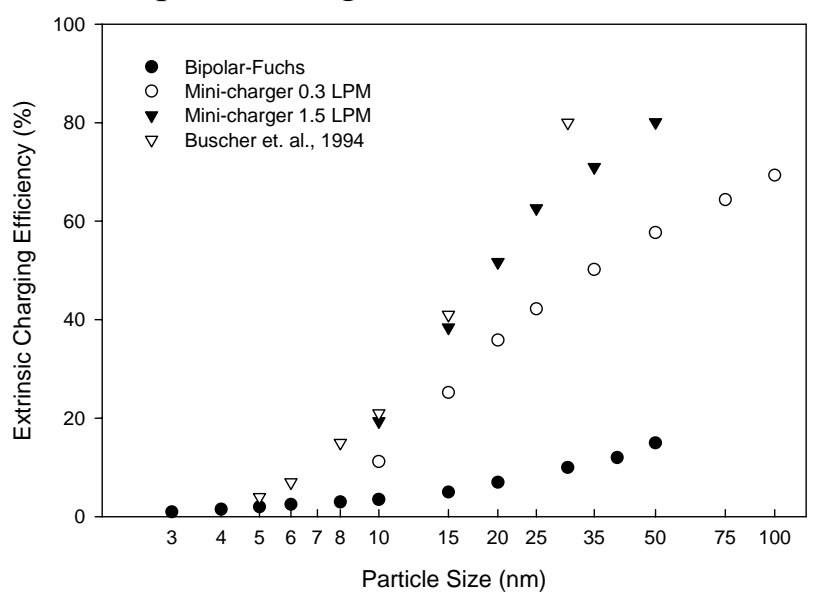

Figure 10: Extrinsic charging performance

Particles occupying the larger fraction of the respiratory range $(0.2-25$ micrometers $)$ are accessible by optical techniques due to their significantly larger scattering cross sections. NASA has developed a miniature sensor for this purpose, arising from requirements associated with both early warning fire detection, and environmental monitoring requirements in support of other government agencies. The package takes advantage of recent advances in semiconductor sources and detectors developed primarily for the telecommunication and media storage sectors. A prototype of a complete integrated package is shown in figure 11. The complete capability includes the drive circuitry for the semiconductor laser, and an imbedded processor to implement calibration constants, data analysis, and internal system checks. Also included are provisions for wireless data transmission.

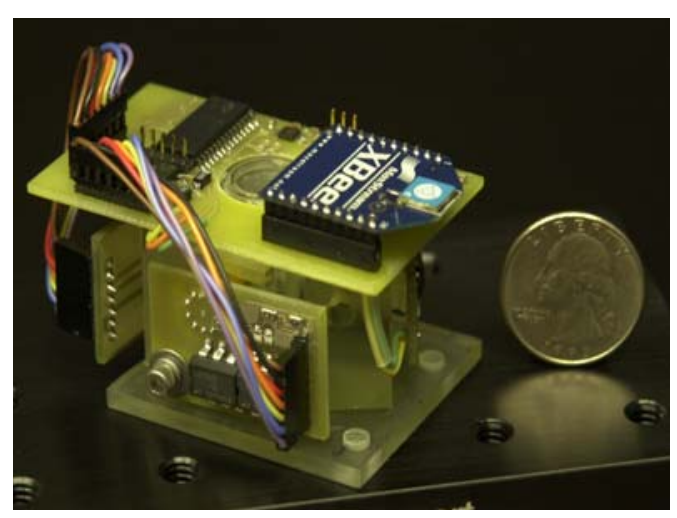

Figure 11: Integrated scattering sensor package

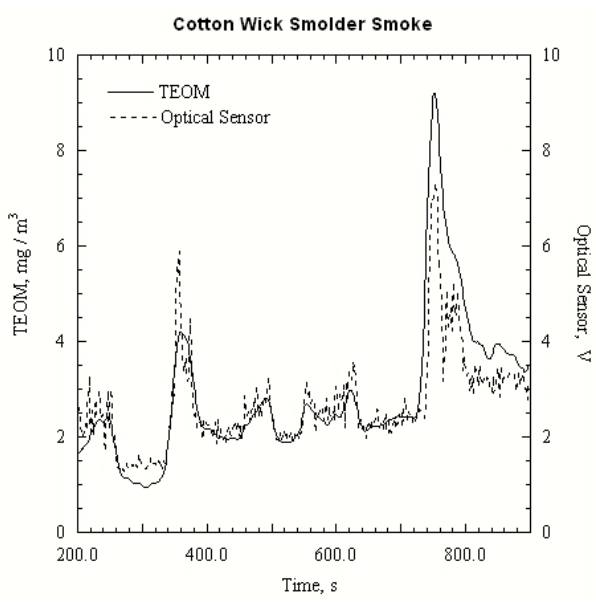

Figure 12: Sensor mass response 
Figure 12 illustrates the sensor response, showing the internally derived output in terms of mass response. Also shown is the data from a reference tapered element oscillating microbalance (TEOM) sampling in parallel. A combination of sensors allows the computation of other moment distributions.

\section{Conclusion}

Planned Lunar missions of extended duration have prompted renewed attention to features of surface regolith, particularly the finest size fractions. The derivation of suitably tolerant component and system designs requires a more complete understanding of this material and its properties. In turn, this creates a demand of sensor technologies for both fundamental measurements and in-situ applications. Described here are provisions for the measurement of PSDs in the fine and ultrafine regimes, as well as techniques for sample fractionation. The latter is motivated by the need for supporting analysis of size-dependent properties, as well as for reverse engineering of bulk materials. A description is also provided of efforts in the development of particulate environmental sensors for end-use mission applications. These efforts are required since existing technologies are insufficient in complying to the associated constraints. Presented are both sensor technologies for the measurement of ultrafines via mobility classification, as well as optical scattering devices to address the larger sizes in the respiratory range.

\section{References}

1. Heiken, G. H., D.T. Vaniman and B. M. French, Lunar Sourcebook (1991), Lunar Planetary Institute, Houston, TX, 285-356.

2. McKay, D. S., et al. (1972), Proc. Lunar Sci. Conf. 3rd, 983.

3. Baron, P. A. and K. Willeke (2001), Aerosol Measurements, Wiley, New York, ed. 2, 508-519, 537-564.

4. Hinds, W. C. (1999), Aerosol Technology, John Wiley \& Sons, New York, 341-345.

5. Liu, B. Y. H. and D. Y. H. Pui (1986), Aerosol charge neutralization by a radioactive alpha source, Part. Charact. 3, 111-116.

6. B. T. Chen, Y. S. Cheng, and H. C. Yeh (1985), Performance of a TSI aerodynamic particle sizer, Aerosol Sci. and Tech., 4, 89-97.

7. B. Y. H. Liu, K. T. Whitby and H. S. Yu (1967), Review of Sci. Instruments, 38(1):100-102.

8. D. B. Blackford and K. L. Rubow (1986), TIZ-Fachberiche, 110 (10), 645-655.

9. http://hefd.jsc.nasa.gov/toxladtag.htm

10. Qui, C., D. Chen and P. S. Greenberg, "Development of a miniaturized nanoparticle sizer for personal exposure study, Part I: Mini-disk electrostatic aerosol classifier," accepted for publication in J. Aeros. Sci.

11. Qui, C., D. Chen and P. S. Greenberg, "Development of a miniaturized nanoparticle sizer for personal exposure study, Part II, Unipolar aerosol mini-charger," accepted for publication in Aeros, Sci. and Tech. 